\title{
Emergency Front of Neck Access
}

\author{
Martin Ince, Venkata Krishnakar Melachuri \\ Department of Anaesthesia, Tameside General Hospital and Integrated Care NHS Foundation Trust, United Kingdom
}

\section{Abstract}

The "Can't Intubate, Can't Oxygenate" (CICO) situation, while rare, is one of the most emergent and stressful scenarios ever faced by airway practitioners. Failure to provide adequate oxygenation can rapidly result in hypoxic brain injury and death. Emergency front of neck access provides a last resort, lifesaving route for the invasive oxygenation of patients. Adequate forward planning as well as recognition of at-risk patients is critical to avoidance of CICO situations. Multiple strategies exist for performing emergency front of neck access, and much debate exist as to which strategy is superior. All airway practitioners should be trained in at least one method of emergency front of neck access, as it may be required in unfamiliar environments at any time. A thorough understanding of the anatomy involved is important to avoid complications, and regular training has been shown to be vital to the maintenance of the skill. It is often the case that front of neck access is performed too late and a great emphasis has been placed on promoting a timely performance of the procedure.

Keywords: Cricothyrotomy, emergency airway, front of neck access

\section{INTRODUCTION}

Emergency front of neck access, nowadays near synonymous with emergency cricothyroidotomy, is a temporary, lifesaving procedure performed as a last resort to provide oxygenation to patients in "can't Intubate, can't Oxygenate" (CICO) situations. ${ }^{[1]}$ The CICO scenario is usually described as when attempts at facemask and supraglottic airway device (SAD) ventilation have failed in a patient who initially had failed attempts at endotracheal intubation. ${ }^{[2]}$ While the term can't intubate, can't ventilate is often used interchangeably with $\mathrm{CICO}, \mathrm{CICO}$ is seen as being a more accurate term, ${ }^{[3]}$ given that it is failure to oxygenate rather than failure to ventilate that will kill the patient in an airway emergency. Occurrences of CICO are thankfully rare, however this has led to problems with familiarity among anesthetists, and delays in performing emergency front of neck access do occur, often with dire consequences. ${ }^{[4]}$ In this review article, we will review the anatomy relevant to emergency cricothyroidotomy, then move on to cover the different techniques available, the problems associated with them, and the evidence basis for their usage.

Cricothyroidotomy can be traced back to at least the early $19^{\text {th }}$ century, when a French anatomist and surgeon named Félix Vicq-d'Azyr ${ }^{[5]}$ described a technique for gaining access to the trachea. It was not however for another 100 years before

\begin{tabular}{|l|l|}
\hline \multicolumn{2}{|c|}{ Access this article online } \\
\hline Quick Response Code: & Website: \\
\hline & www.ijrconline.org \\
\cline { 2 - 2 } & \\
\hline
\end{tabular}

Chevalier Jackson, a laryngologist working in Philadelphia in 1909, described a technique that resembles anything we see today. ${ }^{[6]}$ However, high complication rates - particularly of subglottic stenosis - were later seen which led to Jackson's condemnation. The technique remained out of favor until its revival in the 1970s as an elective technique for long-term ventilation ${ }^{[7]}$ and its potential as a lifesaving maneuver only just beginning to be understood. ${ }^{[8-10]}$ Since then, the technique has been further refined and technology has advanced significantly to better facilitate both elective and emergency use of cricothyroidotomy.

It has been identified that the vast majority of patients who go on to develop hypoxic brain injury as a result of anesthetic airway emergencies pass through a CICO stage. ${ }^{[11,12]}$ Traditional rescue techniques included emergency surgical tracheostomy, however it is now well described in the literature that, even in experienced hands, most will take longer than 3 min to intubate the trachea. ${ }^{[11,13]}$ Often there will be further delay, something that invariably leads to poor outcomes. ${ }^{[14,15]}$ Evidences from organ donors show that cardiac arrest typically occurs within

Address for correspondence: Dr. Venkata Krishnakar Melachuri, Tameside General Hospital and Integrated Care NHS Foundation Trust, United Kingdom. E-mail: mkrishnakar@gmail.com

This is an open access article distributed under the terms of the Creative Commons Attribution-NonCommercial-ShareAlike 3.0 License, which allows others to remix, tweak, and build upon the work non-commercially, as long as the author is credited and the new creations are licensed under the identical terms.

For reprints contact: reprints@medknow.com

How to cite this article: Ince M, Melachuri VK. Emergency front of neck access. Indian J Respir Care 2017;6:793-9. 
5-10 min of anoxia. ${ }^{[2]}$ Clearly this cannot be relied upon in case of emergency, not least because the expertise required may not be at hand.

CICO situations are among the most stressful moments in the careers of anesthetists, or indeed any clinician managing an airway. The incidence of CICO does vary in literature from approximately $1: 6000^{[16]}$ to a perhaps slightly less alarming 1:13000. ${ }^{[17]}$ This does still mean that, in even smaller centers performing 5000 surgeries/year, a case of CICO would be expected once every 3 years. Rates of front of neck access again vary in literature, but are approximately 1:50000 anesthetics. ${ }^{[2]}$ In the emergency department, rates of CICO can reach as high as 1:100. ${ }^{[18]}$ The National Audit Project 4 (NAP4) study, ${ }^{[2]}$ carried out by the Royal College of Anaesthetists, identified 133 serious airway complications (from a total pool of almost 3 million anesthetics) over the study period; 58 had an attempt at emergency front of neck access and of these there were 6 deaths.

Prediction of the CICO situation is seen as being key to its avoidance, by allowing for planning of alternative strategies for airway management. While several prediction tools and grades for the prediction of difficult intubation (Mallampati score, etc.) exist, there are no established grading systems for the prediction of difficult ventilation. ${ }^{[17]}$ While prior difficult intubation or ventilation is seen as the most reliable indicator for future difficulties, clearly this cannot be solely relied upon. A study by Kheterpal et al. ${ }^{[17]}$ established that there is a significant overlap between patients who are difficult to intubate and difficult to ventilate - importantly in patients graded Mallampati III and IV, there are some additional factors which must be taken into account. In most cases, a difficult ventilation is likely to be more problematic than difficult intubation and so it is important that these are recognized. Patients with body mass index of 30 or greater - particularly when distributed around the neck - a history of OSA, limited or severely limited jaw protrusion, and the presence of a beard were all shown to be independent risk factors for difficult or impossible mask ventilation.

\section{Anatomy of the Cricothyroid Membrane}

To perform emergency front of neck access, it is of vital importance that the operator should know the local anatomy relevant to the procedure. ${ }^{[19,20]}$ The avascular cricothyroid membrane is bordered superiorly by the thyroid cartilage (the Adam's apple) and inferiorly by the cricoid cartilage. It is overlain by the pretracheal fascia as well as the cricothyroid arteries and veins, which typically anastomose in the apical portion of the membrane. ${ }^{[21,22]}$ The membrane itself varies in size with age; however in adults, it is approximately $10 \mathrm{~mm}$ in height and $22 \mathrm{~mm}$ across. ${ }^{[23]}$ The cricothyroid muscles overly the edges of the membrane (typically reducing the accessible width to $8.2 \mathrm{~mm}^{[24]}$ ) and the thyroid gland lies adjacent to the lower three edges of the membrane. It is highly unlikely during the procedure for emergency front of neck access that the vocal cords themselves are damaged, as these commonly lie approximately $10 \mathrm{~mm}$ above the cricothyroid membrane. ${ }^{[23]}$

Anatomy is most easily felt with the patients' neck in full extension, allowing for a laryngeal handshake to be performed. ${ }^{[25]}$ The laryngeal handshake is performed by placement of the user's nondominant hand around the larynx, stabilizing it, while using the index finger of that hand to palpate first the thyroid cartilage, and then the cricothyroid membrane itself. In practice, it can often be difficult to palpate the relevant anatomy (perhaps due to the presence of large amounts of subcutaneous fascia), particularly in an emergency situation, and evidence suggests that anesthetists are poor at identifying the cricothyroid membrane in emergency situations. ${ }^{[26-28]}$ Different approaches exist to address this. The Difficult Airway Society Guidelines suggest that a $10 \mathrm{~cm}$ vertical incision should be made along the neck with blunt dissection until the anatomy is seen. ${ }^{[25]}$ Melker and Kost ${ }^{[29]}$ suggested that, by placing the little finger of the right hand on the patients' suprasternal notch with the head in the neutral position and resting the other fingers down together, the resting point of the index finger will usually be over the cricothyroid membrane.

While it must be stressed that it is not recommended for use in the emergency situation, it is becoming more popular to use ultrasound prior to induction to precisely locate the cricothyroid membrane. ${ }^{[30,31]}$ The technique should be performed with the patient's neck in full extension (as would be the case in the emergency situation) to prevent distortion of the skin overlying the structures with neck movement. A transverse plane approach is generally favored moving cranially from the lower tracheal rings in the neck, until the cricothyroid membrane is visualized. In the sagittal plane, a typical appearance of a string of pearls is seen created by the hypoechoic cartilage "pearls" on the hyperechoic air-tissue interface. Once identified, the site of the cricothyroid membrane can be marked with a pen for later use, should the need arise.

\section{Techniques for Emergency Front of Neck Access}

It is important to understand that classical teaching stipulates that cricothyroidotomy by any technique is only a temporizing measure ${ }^{[32]}$ designed to buy time prior to a more definitive airway being established. There is some debate within literature as to the benefits of conversion to tracheostomy, particularly in the case of surgical cricothyroidotomy, however standard practice remains to convert within $24-72 \mathrm{~h}^{[33,34]}$

Several techniques have been described for cricothyroidotomy, and much debate exist as to which technique is superior. ${ }^{[11,25,35]}$ It is unanimously reported, however that regardless of technique, emergency cricothyroidotomy is attempted too late. ${ }^{[4]}$ Most countries have developed their own guidelines for the management of CICO, including emergency front of neck access, such as the Difficult Airway Society Guidelines in the UK. ${ }^{[25]}$ Often the favored technique for such guidelines is based on human factors surrounding the technique rather 
than technical superiority of a technique, something we will discuss in more detail later.

\section{Needle Cricothyroidotomy}

Following palpation of the cricothyroid membrane as per the laryngeal handshake above, a large bore intravenous (IV) cannula or a specifically designed kink-resistant cricothyroidotomy cannula (such as a Patil [Cook] or Ravussin [VBM] needle) is introduced into the membrane at a $45^{\circ}$ caudal angle with negative pressure aspiration through a syringe ${ }^{[3]}$ which may be partially filled with saline. Once the needle is inserted through the membrane (often with a distinct "give" in resistance), air (bubbles) will be freely seen being drawn into the syringe. The cannula should be advanced and the needle must be withdrawn. Further aspiration should take place to reconfirm placement in the trachea. The cannula should be secured, taking care to avoid kinking of the cannula which may impede ventilation attempts, something commonly seen when IV cannulas are employed. ${ }^{[36,37]}$ A means of oxygenation should then be attached to the cannula.

Regardless of the mode of oxygenation, care should be taken to allow sufficient expiration so as to prevent barotrauma and subsequent cardiovascular compromise. ${ }^{[38]}$ Surgical emphysema is not uncommon with this technique. A patent upper airway is mandatory to allow adequate expiration and it is recommended that neuromuscular blockade should be considered at least to prevent laryngospasm as a reason for upper airway obstruction. ${ }^{[3]}$ Airway adjuncts may be used to facilitate opening of the upper airways. Needle cricothyroidotomy is not recommended in the case of upper airway obstruction due to flow limitations through the cannula, preventing adequate expiration within a sufficient time frame to allow adequate minute volumes. This presents a limitation in the technique for the prevention of hypercapnia, which can be catastrophic in head injury. ${ }^{[35]}$ Some have suggested that insertion of a second cannula may assist expiration, ${ }^{[12]}$ however the diameter is felt to be too small to be of real benefit, given the low driving pressure of expiration. ${ }^{[39]}$ It has been suggested that vacuum suction to facilitate active expiration may be of benefit ${ }^{[40]}$ and indeed this has been incorporated into the commercially available Ventrain ${ }^{\circledR}$ system (Ventinova), although studies covering its clinical efficacy are still lacking.

Typically, for narrow-bore cricothyroidotomy cannulas, transtracheal jet insufflators are employed to deliver both high flow and high pressure to the patient, to overcome the resistance of the cannula. Such devices include the Manuje ${ }^{\mathrm{TM}}$ which is capable of delivering pressures from 50 to $400 \mathrm{kPa}$.

Reports of successful ventilation with lower flow systems of sufficient pressure (e.g., wall oxygen supply or cylinder oxygen at a flow rate of $15 \mathrm{~L} / \mathrm{min}$ or greater) exist, ${ }^{[41,42]}$ however often equipment is self-assembled and as such is off license. There is a potential for danger in such situations due to the potential for ineffective devices being assembled. ${ }^{[43]}$ The Enk oxygen flow modulator (Cook) is a simple device designed to function from either piped wall oxygen or a cylinder and has been shown in animal models to match the Manujet ${ }^{\mathrm{TM}}$ for re-oxygenation. ${ }^{[44]}$

Care must be taken with nomenclature as pointed out by Cook et al..$^{[45]}$ as often wall oxygen and cylinder oxygen are erroneously referred to as "low pressure" systems (in the context of "high pressure" systems such as the Manujet thM $^{\mathrm{TM}}$ when in fact their driving pressures are comparable and even greater than that of the Manujet ${ }^{\mathrm{TM}}(400 \mathrm{kPa}$ and $13700 \mathrm{kPa}$, respectively) and it is only their flow rates which differ. This is of crucial significance in the case of attempted transtracheal ventilation from the common gas outlet of an anesthetic machine. The back bar of the anesthetic machine has a safety "blow out" valve that prevents delivery of pressures exceeding 40-50 $\mathrm{kPa}$ and consequently this has been shown to be an ineffective source for delivery of oxygen through a cricothyroidotomy cannula. ${ }^{[46,47]}$ In the case of a self-inflating bag or anesthetic breathing circuit, this inadequacy is made further worse and should not be used. Furthermore, the often used terminology "jet ventilator" may be misleading, given that the typically high frequency and transtracheal pressures generated are somewhat different to conventional ventilation, and instead the terminology "jet insufflator" is preferable.

It is becoming increasingly commonplace for anesthetists to place an awake cricothyroidotomy cannula prior to induction of anesthesia in patients with an anticipated difficult airway. ${ }^{[23,48]}$ The procedure is well tolerated and easily performed under local anesthetic with or without systemic analgesia. ${ }^{[49]}$ The cannula can then be secured and capped off, and the anesthetist can proceed with induction, safe in the knowledge that, should the need arise, the cannula is already in place. Typically, the cannula remains in place until after successful extubation.

Wide bore cannula over trocar techniques do exist, such as in the case of the Portex ${ }^{\circledR}$ cricothyroidotomy or the Quicktrach II (VBM). These have the advantage of being able to place a wide bore tube (typically of internal diameter $>4 \mathrm{~mm}$ ), however insertion requires considerable force and risks damage to the posterior trachea $\mathrm{a}^{[50]}$ - something shown to occur almost $70 \%$ of the time in an animal model. ${ }^{[5]]}$ NAP4 noted that the safety mechanism built into the Quicktrach designed to attempt to reduce the considerable risk of posterior wall trauma made use of the device in the obese neck difficult. ${ }^{[2]}$

\section{Seldinger Cricothyroidotomy}

The Seldinger technique is the one familiar to anesthetists, involving the passage of a guidewire prior to dilation and passage of the tube. Dilation after initial puncture reduces the risk of posterior wall trauma as seen with the cannula-over-trocar techniques. ${ }^{[52]}$ The Portex Mini $\operatorname{Trach}^{\mathrm{TM}}$ is an example of such a device and can be commonly found in hospitals, however it is not licensed or recommended for use in CICO situations. The Melker emergency cricothyroidotomy set is designed for use in such situations, coming with a range of sizes from 3 to $6 \mathrm{~mm}$ internal diameters, and it is generally felt that this technique is most acceptable to anesthetists - most 
likely due to the familiarity with the Seldinger technique. ${ }^{[53]}$ Criticisms of the technique include a long insertion time ${ }^{[54]}$ (although this is disputed), ${ }^{[55,56,]}$ risks for kinking of the guidewire, ${ }^{[57]}$ creation of false passage, and high failure rates in inexperienced hands. ${ }^{[58]}$

\section{Surgical Cricothyroidotomy}

As with percutaneous techniques, surgical cricothyroidotomy begins with palpation of the landmarks, by use of the laryngeal handshake. Techniques and equipment for surgical cricothyroidotomy do differ slightly. The Advanced Trauma Life Support advocates the use of a no. 11 blade scalpel to make an initial horizontal stab incision, followed by blunt dilation either by use of forceps or the blade handle. ${ }^{[59]}$ The Difficult Airway Society, however, truncates the process by first making a horizontal stab incision with a no. 10 blade scalpel and then rotating the blade in situ $90^{\circ}$ caudally, before passing a gum elastic bougie prior to railroading a size 6 internal diameter cuffed endotracheal tube ${ }^{[25]}$ [Figures 1-3]. The no. 10 blade has a curved tip compared to the no. 11 blade's point which has the advantage of reducing the risk for posterior wall trauma. ${ }^{[3]}$ If available, a no. 20 blade may be preferable to a no. 10 blade as its larger size may facilitate easier passage of the ET tube. Some techniques suggest the use of a tracheal hook to keep the incision patent while inserting the tube, ${ }^{[60]}$ however it is recognized that such implements are not widely available in emergency situations and as such more familiar and available equipment such as the gum elastic bougie are used. ${ }^{[25]}$ Insertion of a cuffed tube allows for securing of the airway against aspiration, something not possible with narrow bore needle techniques. Conventional ventilation can be established using standard equipment and monitoring. Catastrophic blood loss is rare when the technique is performed correctly. ${ }^{[3]}$ Endobronchial intubation is a recognized complication from surgical cricothyroidotomy and should be avoided. ${ }^{[61]}$ In the emergency situation of CICO, there are no strict contraindications, however it should be noted that, in the case of the pediatric airway, percutaneous techniques may be more successful, due to anatomical differences ${ }^{[62]}$ though cutoff ages are poorly defined.

Numerous studies have suggested that scalpel cricothyroidotomy is the most reliable and fastest of the emergency front of neck access techniques. ${ }^{[63-65]}$ Evidence also suggests that the actual time difference between performance of either needle or scalpel cricothyroidotomy is seconds rather than minutes. ${ }^{[54]}$ When weighed against other factors, there is no good evidence to suggest that this small time factor grossly impacts mortality.

\section{Problems Inherent to Emergency Front of Neck AcCESS}

The American Society of Anesthesiologists closed claims report showed that, when front of neck access was attempted, it was attempted too late in as many as $70 \%$ of cases. ${ }^{[4]}$ Tragically this was seen in the case of Elaine Bromiley, who died as a result of a $\mathrm{CICO}$ situation. Criticism was made that, despite

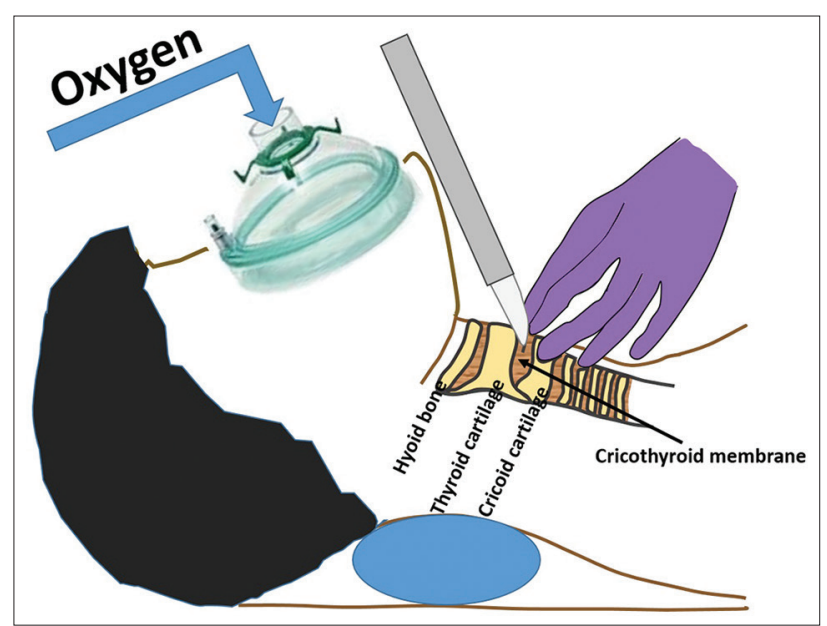

Figure 1: Incision of cricothyroid membrane

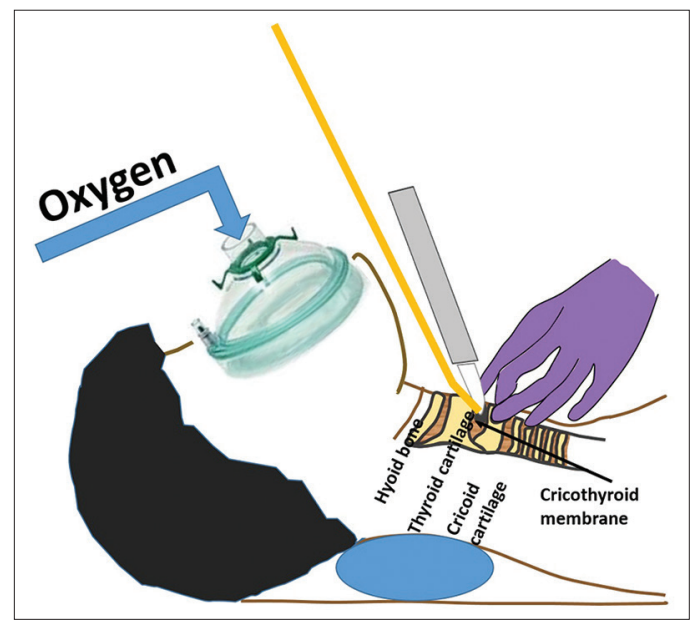

Figure 2: Introduction of bougie

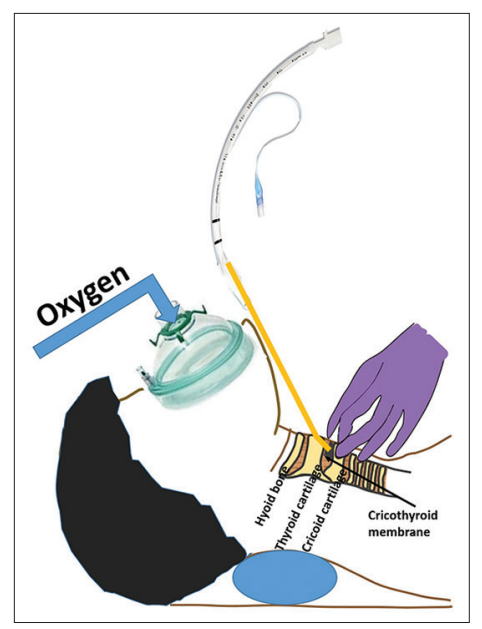

Figure 3: Railroading the endotracheal tube

impossible mask ventilation for nearly $30 \mathrm{~min}$ and failure to rescue with a SAD, no attempt was made at cricothyroidotomy, and persistent failed attempts at laryngoscopy were made. ${ }^{[66]}$ There have been numerous reasons postulated for why delays 
occur, however clearly reducing this figure would appear to dwarf technical superiority differences, which may only vary in seconds to minutes.

There is a great deal of reluctance among anesthetists to take a scalpel to a patient's neck, whereas it would seem as a profession anesthetists are far more comfortable making use of needle or Seldinger techniques. In a survey conducted by Wong et al. in 2014, just 3\% of anesthetists would choose a scalpel-bougie as a first choice technique, increasing to a mere $7 \%$ for second choice. By comparison, 39\% would choose a Seldinger technique and $28 \%$ would choose an IV cannula technique. ${ }^{[53]}$ Clearly, devices well known to anesthetists - IV cannulas and Seldinger-based kits, have a number of transferrable skills, perhaps decreasing the delay in attempting cricothyroidotomy. ${ }^{[25]}$

Chrimes suggests that, rather than a rigid guideline for the management of a difficult airway, a more fluid vortex approach should be considered. ${ }^{[67]}$ The vortex consists of strategies for achieving ventilation around outside with a final central point of emergency front of neck access. The idea behind the vortex is that, as hypoxia develops, so are time and options limited, encouraging efficient progression through techniques and timely progression to front of neck access. The inclusion of the three-dimensional vortex reminds us that, as techniques are attempted, they are not returned to.

The CICO situation is thankfully rare, ${ }^{[16,17]}$ however this does present challenges in ensuring clinicians remain up to date and well practiced with devices. As has been previously discussed, there are multiple techniques and many more different pieces of equipment available which may differ in their availability between centers. Some countries, including the UK, have focused their efforts on standardizing techniques and equipment, thereby reducing the requirement for clinicians to learn multiple techniques, as well as removing any decision-making from the process as to which technique to use. It is well understood that regular training is required to maintain the performance of emergency front of neck access. ${ }^{[68]}$ It is recommended that practice of the technique by means of simulation is undertaken at least once per 6 months to maintain skill, ${ }^{[69]}$ however it has been shown that the closer to the time of the last practice is predictive of performance, ${ }^{[70]}$ and perhaps an even shorter time interval between practices is beneficial.

\section{Postprocedure Care}

Clear and precise documentation is essential after any airway emergency, particularly so in the case of emergency cricothyroidotomy. The patient should be counseled regarding the future procedures and all steps taken to ensure prior planning in the future. In the UK, there is a standardized alert pro forma for the difficult airway, produced by the Difficult Airway Society ${ }^{[71]}$ to assist with communication, recognized often to be poor. ${ }^{[72,73]}$
In cases where there is no option to wake the patient, conversion to formal tracheostomy may be indicated. A narrow-bore cricothyroidotomy may be converted to a wider bore by the use of the Seldinger cricothyroidotomy kit. ${ }^{[3]}$

It is important that patients who have had airway emergencies are followed up postoperatively by anesthetists, as it is well reported that airway trauma occurs frequently in this group. Laryngeal, pharyngeal, and esophageal injuries were reported to be the most common complications. ${ }^{[74]}$ Only half of the patients with pharyngoesophageal perforation show early signs such as pneumothorax or subcutaneous emphysema. Symptoms of serious complications including retropharyngeal abscess and mediastinitis should be communicated to patients and should be advised accordingly.

Regardless of outcome, airway emergencies can have profound effects on health-care staff. As often patients with airway emergencies are previously healthy patients or even children, serious sequelae including the development of posttraumatic stress disorders, depression, and burnout have been described. ${ }^{[75]}$ An initial debrief following critical incidents is recommended for the entire medical team, to assist with diffusing any tensions within the team, as well as a formal debrief attended by a mental health professional or counselor at a later date. ${ }^{[76]}$ It is often wise to encourage all team members to write a short factual account of the event soon after to ensure that memory of the event and to avoid blurring by discussion with others over their individual accounts.

\section{Conclusion}

It is quite evident that all techniques for emergency front of neck access have pros and cons associated with them. No conclusive evidence exists that favors any one technique to the exclusion of others for solely technical reasons. As previously discussed, training and ongoing practice is essential to maintain the performance of any technique, which improves success rates in emergency front of neck access. In the UK, at present, there is no specific requirement for training in emergency front of neck access as part of ongoing revalidation and as such there needs to be ongoing local efforts for training and reskilling in emergency front of neck access. There must be a great emphasis on reducing delays in the performance of emergency front of neck access to avoid tragedy. As airway emergencies can and do happen anywhere both in and out of hospital, it is essential that necessary equipment is available in multiple locations. Such arguments favor a single technique, as per the UK, with the additional benefit of removing a stage of decision-making from the user. Regardless of technique, human factors will invariably play a large role in airway emergencies, and when developing guidelines, this must be taken into account.

\section{Financial support and sponsorship}

Nil.

\section{Conflicts of interest}

There are no conflicts of interest. 


\section{RefEREnCES}

1. Timmermann A, Chrimes N, Hagberg CA. Need to consider human factors when determining first-line technique for emergency front-of-neck access. Br J Anaesth 2016;117:5-7.

2. $4^{\text {th }}$ National Audit Project of the Royal College of Anaesthetists, 2011. Available from: http://www.rcoa.ac.uk/document-store/nap4-fullreport. [Last accessed on 2017 Jun 27].

3. Hamaekers AE, Henderson JJ. Equipment and strategies for emergency tracheal access in the adult patient. Anaesthesia 2011;66 Suppl 2:65-80.

4. Metzner J, Posner KL, Lam MS, Domino KB. Closed claims' analysis. Best Pract Res Clin Anaesthesiol 2011;25:263-76.

5. GovenderV. Cricothyroidotomy and the DifficultAirway;2016. Available from: https://www.google.co.uk/url? sa $=t \& r c t=j \& q=\&$ esrc $=$ s\&source $=$ web\&cd $=4 \& \mathrm{cad}=$ rja\&uact $=8 \& v e d=0$ ahUKEwit $2 \mathrm{PuV} 4 \mathrm{~N} 3 \mathrm{UAhXR}$ U1AKHRr4CfoQFgg2MAM\&url=http $\% 3 \mathrm{~A} \% 2 \mathrm{~F} \% 2 \mathrm{~F}$ anaesthetics. ukzn.ac.za\%2FLibraries\%2FICU_1\%2FFMM_No_24_14-10-2016_ Cricothyroidotomy_and_the_Difficult_Airway_V_Govender.sflb.ashx \&usg=AFQjCNEbYSzzSJNRjUITQTxCRwuEkg-uZg [Last accessed on 2017 Jun 27].

6. Jackson C. Tracheotomy. Laryngoscope 1909;18:285.

7. Brantigan CO, Grow JB Sr. Cricothyroidotomy: Elective use in respiratory problems requiring tracheotomy. J Thorac Cardiovasc Surg 1976;71:72-81.

8. Hammer WB. The cricothyroidotomy - A lifesaving adjunct in acute obstructive asphyxia. J Ga Dent Assoc 1969;43:35-8.

9. Haber H. Difficult intubations. Middle East J Anaesthesiol 1979;5:195-201.

10. Boyd AD, Romita MC, Conlan AA, Fink SD, Spencer FC. A clinical evaluation of cricothyroidotomy. Surg Gynecol Obstet 1979;149:365-8.

11. Henderson JJ, Popat MT, Latto IP, Pearce AC; Difficult Airway Society. Difficult Airway Society guidelines for management of the unanticipated difficult intubation. Anaesthesia 2004;59:675-94.

12. Jacobs HB. Emergency percutaneous transtracheal catheter and ventilator. J Trauma 1972;12:50-5.

13. Finucane B. The difficult airway - A Canadian perspective. Can J Anaesth 1998;45:713-8.

14. Suh RH, Margulies DR, Hopp ML, Ault M, Shabot MM. Percutaneous dilatational tracheostomy: Still a surgical procedure. Am Surg 1999;65:982-6.

15. Griggs WM, Myburgh JA, Worthley LI. Urgent airway access - An indication for percutaneous tracheostomy? Anaesth Intensive Care 1991;19:586-7.

16. Nagaro T, Yorozuya T, Sotani M, Adachi N, Tabo E, Arai T, et al. Survey of patients whose lungs could not be ventilated and whose trachea could not be intubated in university hospitals in Japan. J Anesth 2003; 17:232-40.

17. Kheterpal S, Martin L, Shanks AM, Tremper KK. Prediction and outcomes of impossible mask ventilation: A review of 50,000 anesthetics. Anesthesiology 2009;110:891-7.

18. Chang RS, Hamilton RJ, Carter WA. Declining rate of cricothyrotomy in trauma patients with an emergency medicine residency: Implications for skills training. Acad Emerg Med 1998;5:247-51.

19. Burkey B, Esclamado R, Morganroth M. The role of cricothyroidotomy in airway management. Clin Chest Med 1991;12:561-71.

20. Bennett JD, Guha SC, Sankar AB. Cricothyrotomy: The anatomical basis. J R Coll Surg Edinb 1996;41:57-60.

21. Boon JM, Abrahams PH, Meiring JH, Welch T. Cricothyroidotomy: A clinical anatomy review. Clin Anat 2004;17:478-86.

22. Little CM, Parker MG, Tarnopolsky R. The incidence of vasculature at risk during cricothyroidostomy. Ann Emerg Med 1986;15:805-7.

23. Goon SS, Stephens RC, Smith H. The emergency airway. Br J Hosp Med (Lond) 2009;70:M186-8.

24. Dover K, Howdieshell TR, Colborn GL. The dimensions and vascular anatomy of the cricothyroid membrane: Relevance to emergent surgical airway access. Clin Anat 1996;9:291-5.

25. Frerk C, Mitchell VS, McNarry AF, Mendonca C, Bhagrath R, Patel A, et al. Difficult Airway Society 2015 guidelines for management of unanticipated difficult intubation in adults. $\mathrm{Br} \mathrm{J}$ Anaesth $2015 ; 115: 827-48$
26. Lamb A, Zhang J, Hung O, Flemming B, Mullen T, Bissell MB, et al. Accuracy of identifying the cricothyroid membrane by anesthesia trainees and staff in a Canadian institution. Can J Anaesth 2015;62:495-503.

27. Kristensen MS, Teoh WH, Rudolph SS, Tvede MF, Hesselfeldt R, Børglum J, et al. Structured approach to ultrasound-guided identification of the cricothyroid membrane: A randomized comparison with the palpation method in the morbidly obese. Br J Anaesth 2015;114:1003-4.

28. Aslani A, Ng SC, Hurley M, McCarthy KF, McNicholas M, McCaul CL. Accuracy of identification of the cricothyroid membrane in female subjects using palpation: An observational study. Anesth Analg 2012;114:987-92.

29. Melker RJ, Kost KM. Percutaneous dilational cricothyroidotomy and tracheostomy. In: Hagberg C, editor. Benumof's Airway Management. $2^{\text {nd }}$ ed. Mosby: Elsevier, Philadelphia; 2007. p. 640-77.

30. Mallin M, Curtis K, Dawson M, Ockerse P, Ahern M. Accuracy of ultrasound-guided marking of the cricothyroid membrane before simulated failed intubation. Am J Emerg Med 2014;32:61-3.

31. Siddiqui N, Arzola C, Friedman Z, Guerina L, You-Ten KE. Ultrasound improves cricothyrotomy success in cadavers with poorly defined neck anatomy: A randomized control trial. Anesthesiology 2015;123:1033-41.

32. Talving $\mathrm{P}$, DuBose J, Inaba $\mathrm{K}$, Demetriades $\mathrm{D}$. Conversion of emergent cricothyrotomy to tracheotomy in trauma patients. Arch Surg 2010;145:87-91.

33. Macêdo MB, Guimarães RB, Ribeiro SM, Sousa KM. Emergency cricothyrotomy: Temporary measure or definitive airway? A systematic review. Rev Col Bras Cir 2016;43:493-9.

34. Graham DB, Eastman AL, Aldy KN, Carroll EA, Minei JP, Brakenridge SC, et al. Outcomes and long term follow-up after emergent cricothyroidotomy: Is routine conversion to tracheostomy necessary? Am Surg 2011;77:1707-11.

35. Scrase I, Woollard M. Needle vs. surgical cricothyroidotomy: A short cut to effective ventilation. Anaesthesia 2006;61:962-74.

36. Metz S, Parmet JL, Levitt JD. Failed emergency transtracheal ventilation through a 14-gauge intravenous catheter. J Clin Anesth 1996;8:58-62.

37. Sdrales L, Benumof JL. Prevention of kinking of a percutaneous transtracheal intravenous catheter. Anesthesiology 1995;82:288-91.

38. Craven RM, Vanner RG. Ventilation of a model lung using various cricothyrotomy devices. Anaesthesia 2004;59:595-9.

39. Morley D, Thorpe CM. Apparatus for emergency transtracheal ventilation. Anaesth Intensive Care 1997;25:675-8.

40. Dunlap LB, Oregon E. A modified, simple device for the emergency administration of percutaneous transtracheal ventilation. JACEP 1978;7:42-6.

41. Bould MD, Bearfield P. Techniques for emergency ventilation through a needle cricothyroidotomy. Anaesthesia 2008;63:535-9.

42. Schaefer R, Hueter L, Preussler NP, Schreiber T, Schwarzkopf K. Percutaneous transtracheal emergency ventilation with a self-made device in an animal model. Paediatr Anaesth 2007;17:972-6.

43. Tighe SQ, Staber M, Hardman JG, Henderson JJ. Emergency airway access equipment. Anaesthesia 2004;59:505-6.

44. Yildiz Y, Preussler NP, Schreiber T, Hueter L, Gaser E, Schubert H, et al. Percutaneous transtracheal emergency ventilation during respiratory arrest: Comparison of the oxygen flow modulator with a hand-triggered emergency jet injector in an animal model. Am J Emerg Med 2006;24:455-9.

45. Cook TM, Nolan JP, Magee PT, Cranshaw JH. Needle cricothyroidotomy. Anaesthesia 2007;62:289-90.

46. Ryder IG, Paoloni CC, Harle CC. Emergency transtracheal ventilation: Assessment of breathing systems chosen by anaesthetists. Anaesthesia 1996;51:764-8.

47. Meyer PD. Emergency transtracheal jet ventilation system. Anesthesiology 1990;73:787-8.

48. Teo N, Garrahy A. Elective surgical cricothyroidotomy in oral and maxillofacial surgery. Br J Oral Maxillofac Surg 2013;51:779-82.

49. Gerig HJ, Schnider T, Heidegger T. Prophylactic percutaneous transtracheal catheterisation in the management of patients with anticipated difficult airways: A case series. Anaesthesia 2005;60:801-5.

50. Abbrecht PH, Kyle RR, Reams WH, Brunette J. Insertion forces and risk of complications during cricothyroid cannulation. J Emerg Med 1992;10:417-26. 
51. Murphy C, Rooney SJ, Maharaj CH, Laffey JG, Harte BH. Comparison of three cuffed emergency percutaneous cricothyroidotomy devices to conventional surgical cricothyroidotomy in a porcine model. Br J Anaesth 2011;106:57-64.

52. Metterlein T, Frommer M, Ginzkey C, Becher J, Schuster F, Roewer $\mathrm{N}$, et al. A randomized trial comparing two cuffed emergency cricothyrotomy devices using a wire-guided and a catheter-over-needle technique. J Emerg Med 2011;41:326-32.

53. Wong DT, Mehta A, Tam AD, Yau B, Wong J. A survey of Canadian anesthesiologists' preferences in difficult intubation and "cannot intubate, cannot ventilate" situations. Can J Anaesth 2014;61:717-26.

54. Johnson DR, DunlapA, McFeeley P, Gaffney J, Busick B. Cricothyrotomy performed by prehospital personnel: A comparison of two techniques in a human cadaver model. Am J Emerg Med 1993;11:207-9.

55. Chan TC, Vilke GM, Bramwell KJ, Davis DP, Hamilton RS, Rosen P. Comparison of wire-guided cricothyrotomy versus standard surgical cricothyrotomy technique. J Emerg Med 1999;17:957-62.

56. Eisenburger P, Laczika K, List M, Wilfing A, Losert H, Hofbauer R, et al. Comparison of conventional surgical versus Seldinger technique emergency cricothyrotomy performed by inexperienced clinicians. Anesthesiology 2000;92:687-90.

57. Schaumann N, Lorenz V, Schellongowski P, Staudinger T, Locker GJ, Burgmann $\mathrm{H}$, et al. Evaluation of Seldinger technique emergency cricothyroidotomy versus standard surgical cricothyroidotomy in 200 cadavers. Anesthesiology 2005;102:7-11.

58. Helm M, Hossfeld B, Jost C, Lampl L, Böckers T. Emergency cricothyroidotomy performed by inexperienced clinicians - Surgical technique versus indicator-guided puncture technique. Emerg Med J 2013;30:646-9.

59. American College of Surgeons Committee on Trauma, Advanced Trauma Life Support Program for Doctors. $9^{\text {th }}$ ed. American College of Surgeons; 2012.

60. Heymans F, Feigl G, Graber S, Courvoisier DS, Weber KM, Dulguerov P. Emergency cricothyrotomy performed by surgical airway-naive medical personnel: A randomized crossover study in cadavers comparing three commonly used techniques. Anesthesiology 2016;125:295-303.

61. Engoren M, de St Victor P. Tension pneumothorax and contralateral presumed pneumothorax from endobronchial intubation via cricothyroidotomy. Chest 2000;118:1833-5.

62. Coté CJ, Hartnick CJ. Pediatric transtracheal and cricothyrotomy airway devices for emergency use: Which are appropriate for infants and children? Paediatr Anaesth 2009;19 Suppl 1:66-76.

63. Lockey D, Crewdson K, Weaver A, Davies G. Observational study of the success rates of intubation and failed intubation airway rescue techniques in 7256 attempted intubations of trauma patients by pre-hospital physicians. Br J Anaesth 2014;113:220-5.

64. Hubble MW, Wilfong DA, Brown LH, Hertelendy A, Benner RW. A meta-analysis of prehospital airway control techniques part II: Alternative airway devices and cricothyrotomy success rates. Prehosp Emerg Care 2010;14:515-30.

65. Mabry RL. An analysis of battlefield cricothyrotomy in Iraq and Afghanistan. J Spec Oper Med 2012;12:17-23.

66. Harmer M. The Case of Elaine Bromiley. 2005. Available from: $\quad$ http://www.chfg.org/wpcontent/uploads/2010/11/ ElaineBromileyAnonymousReport.pdf [Last accessed on 2017 Jun 27].

67. Chrimes N. The Vortex: A universal 'high-acuity implementation tool' for emergency airway management. Br J Anaesth 2016;117 Suppl 1:i20-7.

68. Baker PA, Weller JM, Greenland KB, Riley RH, Merry AF. Education in airway management. Anaesthesia 2011;66 Suppl 2:101-11.

69. Cook TM. (Still) time to organise training in airway management in the UK. Anaesthesia 2006;61:727-30.

70. Kuduvalli PM, Jervis A, Tighe SQ, Robin NM. Unanticipated difficult airway management in anaesthetised patients: A prospective study of the effect of mannequin training on management strategies and skill retention. Anaesthesia 2008;63:364-9.

71. Airway Alert Form. Available from: http://www.das.uk.com/guidelines/ airwayalert.html. [Last accessed on 2017 Jun 27].

72. Wilkes M, Beattie C, Gardner C, McNarry AF. Difficult airway communication between anaesthetists and general practitioners. Scott Med J 2013;58:2-6.

73. Barron FA, Ball DR, Jefferson P, Norrie J. 'Airway alerts'. How UK anaesthetists organise, document and communicate difficult airway management. Anaesthesia 2003;58:73-7.

74. Domino KB, Posner KL, Caplan RA, Cheney FW. Airway injury during anesthesia: A closed claims analysis. Anesthesiology 1999;91:1703-11.

75. Halpern J, Maunder RG, Schwartz B, Gurevich M. Identifying risk of emotional sequelae after critical incidents. Emerg Med J 2011;28:51-6.

76. Aitkenhead AR. Anaesthetic disasters: Handling the aftermath Anaesthesia 1997;52:477-82. 